\title{
Pulmonary Lymphangitic Mesotheliomatosis
}

\section{Pierre Edde*, Nehme Romy, and Chababi Mirna}

Centre Hospitalier Universitaire -Notre Dame De Secours (CHU-NDS) Hospital, Jbeil, Medical school of University Saint Esprit-Kaslik (USEK), Kaslik, Lebanon

\begin{abstract}
We report a 45 year old male with right sided pleural mesothelioma who received neoadjuvant chemotherapy prior to a right extrapleural pneumonectomy followed by adjuvant chemotherapy. The patient became tumor free and remained in remission for 19 months following which he developed a lymphangitic spread over the contralateral lung. His diagnosis was confirmed by a bronchoscopic transbronchial biopsy of the left lung. The patient died from progressive respiratory failure over a period of three months. Such a relapse with an aggressive and fatal lymphangitic spread is rarely seen and reported in malignant pleural mesothelioma. Discussion and review of the literature are provided.
\end{abstract}

Mesothelioma is still considered, worldwide, a rare cancer of the serosal membranes. It typically involves the pleural cavity but other reported sites include the peritoneum, pericardium and Tunica vaginalis of the testis. The usual progression of the mesothelioma is coalescence of multiple, small nodules into large masses that invade, entrap and destroy the affected organ by direct extension and invasion. We report an unusual case of relapsing mesothelioma, recurring in an aggressive and fatal form, after 19 months of remission.

\section{Case Report}

T. H. is a 45 year old man who was exposed to asbestos from his neighborhood between the ages of 6 to 12 years of age. He was diagnosed with mesothelioma in April 2009 by right pleural biopsy. He received four cycles of neoadjuvant chemotherapy followed by right Extrapleural Pneumonectomy (EPP), in June 2009, and then three cycles of adjuvant chemotherapy. He was followed up with serial CT scans of the chest every 6 months. He remained radiographically tumor free for 19 months. On Jan 17, 2011, CT Chest revealed a $2 \mathrm{~cm}$ substernal nodule. At that time, he was completely asymptomatic. Two weeks later, he started complaining of a dry cough refractory to treatment. Open biopsy of the nodule showed only fibrotic tissue. The patient's cough progressed in severity. He became dyspneic to moderate effort. Chest X-Ray followed by CT scan of the Chest confirmed the presence of a severe interstitial process (Figures 1 and 2) proven by a bronchoscopic transbronchial biopsy to be recurrence of the mesothelioma in a lymphangitic pattern. There was no left pleural masses or mediastinal adenopathies noted. The patient expired after nine days from progressive respiratory failure.

\section{Discussion}

Asbestos is a naturally occurring fibrous silicate which was widely used because of its favorable physical and chemical properties such as flexibility, strength, durability and its resistance to heat and acid. However, exposure to asbestos fibers was clearly associated with certain diseases resulting in implementation of strict rules and regulations to avoid any potential hazard from exposure to the asbestos workers, their family members and the general public [1].

Asbestos related diseases vary from asbestosis to lung cancer, benign pleural effusion and thickening, pleural calcification and mesothelioma. Of note that beside the amount and duration of exposure, the time since exposure is an important factor for the appearance of certain diseases: Benign pleural effusion is usually seen between 10 to 20 years with no malignancy diagnosed within 3 years of its appearance. Lung cancer is usually seen after 20 to 30 years while pleural plaques and mesothelioma after 30 years [1] In our case, mesothelioma was diagnosed at the age of 43; i.e., 31 to 37 years since exposure to the asbestos fibers.

Mesothelioma has a poor life expectancy of less than a year. With combined therapeutic modalities using chemotherapy, radiation therapy and surgical resection survival rates have increased. Nevertheless, the overall prognosis is still considered poor. In our case, neoadjuvant and adjuvant chemotherapy were able to maintain the patient disease free for about 19 months following EPP, an outcome that seems superior to the survival rates reported in the review of Sharif et al. [2]. To our knowledge this is the first reported case of aggressive recurrence following a "longer than average" disease-free state and progression from complete symptoms-free and a normal appearing lung parenchyma, to a fatal respiratory failure over less than three months.

Distant metastasis has been reported by Huncharek to occur in $76 \%$ of the patients at autopsy with lung, kidneys, liver and adrenals being the most frequent sites [3]. It is usually late in the disease process and seldom of clinical relevance because of the overall prognosis of the disease and its local destructive pattern. Brain [4], lymphadenopathy $[5,6]$, and isolated pulmonary opacities have been reported. Diffuse and widespread lymphangitic metastasis, whether from the pleural cavity [7] or the peritoneum [8], is a rare occurrence, and is considered to be a distant metastasis rather than direct extension [9]. This is a very aggressive pattern of metastasis resulting in quick progression into respiratory failure and death of all reported cases, similar to our patient.

Extensive and careful review of the literature revealed the report of only 9 cases. We agree with Livasy et al. [10] that the cases reported by Huncharek [3] and Scully [11] are so similar that we also consider them as the same patient. We reviewed the series reported by Nind et al. [12]. Fifty three of their 200 cases had a lymphangitic growth pattern. However, a phone conversation with Dr Attoonas confirmed that their report of lymphangitic growth pattern is a pure pathological diagnosis limited to the biopsy specimens rather than a diffuse lymphangitic involvement of the lung, based on a radiographical diagnosis and meeting the definition of carcinomatosis.

*Corresponding author: Pierre Edde, Notre Dame De Secours (CHU-NDS) Hospital, Jbeil, Medical school of University Saint Esprit-Kaslik (USEK), Kaslik, Lebanon, E-mail: mpedde@yahoo.com

Received February 04, 2012; Accepted February 27, 2013; Published March 04 2013

Citation: Edde P, Romy N, Mirna C (2013) Pulmonary Lymphangitic Mesotheliomatosis. J Pulmon Resp Med 3: 136. doi:10.4172/2161-105X.1000136

Copyright: ( 2013 Edde $P$, et al. This is an open-access article distributed under the terms of the Creative Commons Attribution License, which permits unrestricted use, distribution, and reproduction in any medium, provided the original author and source are credited. 


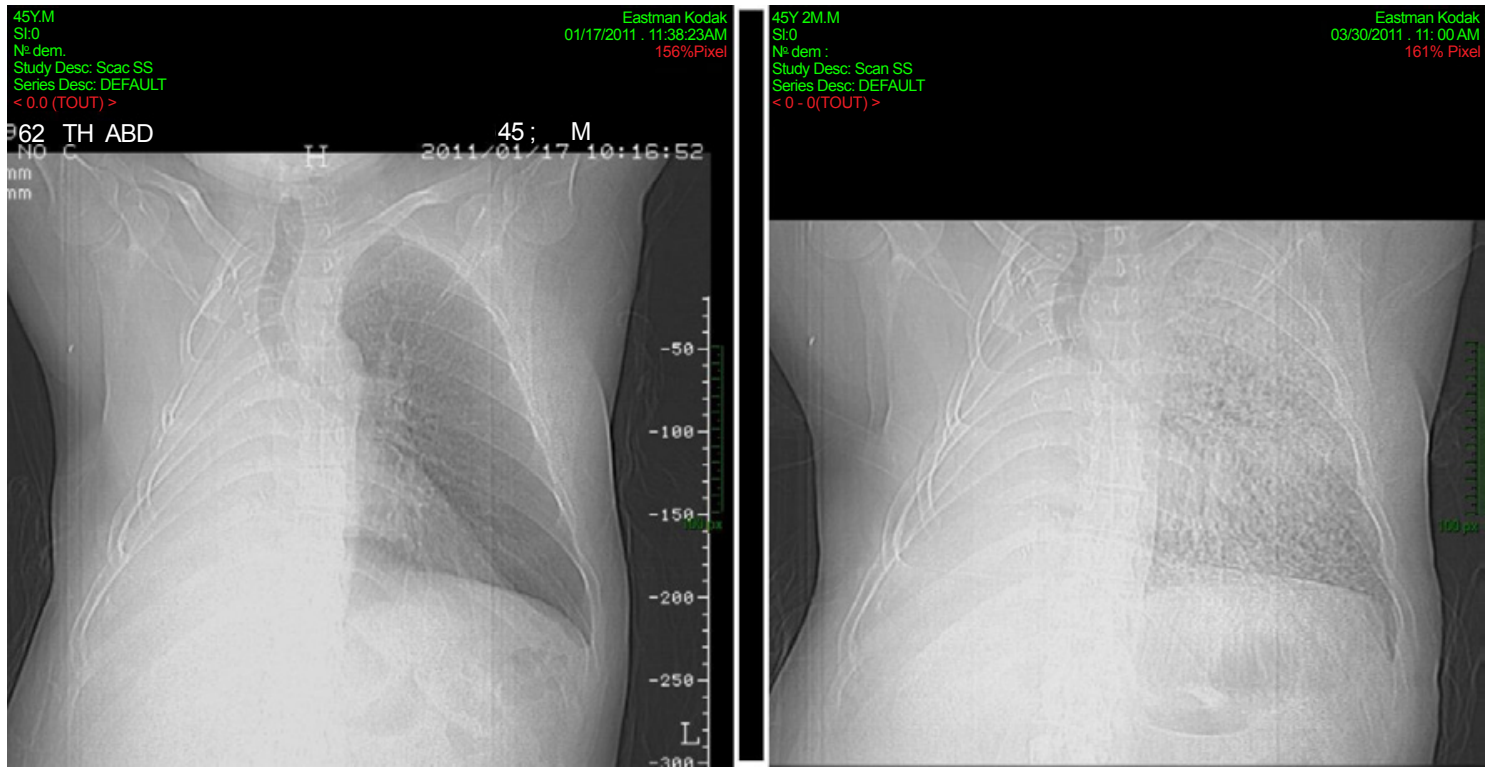

Figure 1: To the left: CXR done on 1/17/2011 (19 months post Right pneumonectomy) showing normal Left lung. To the right: same view after 2.5 months showing diffuse infiltrates.
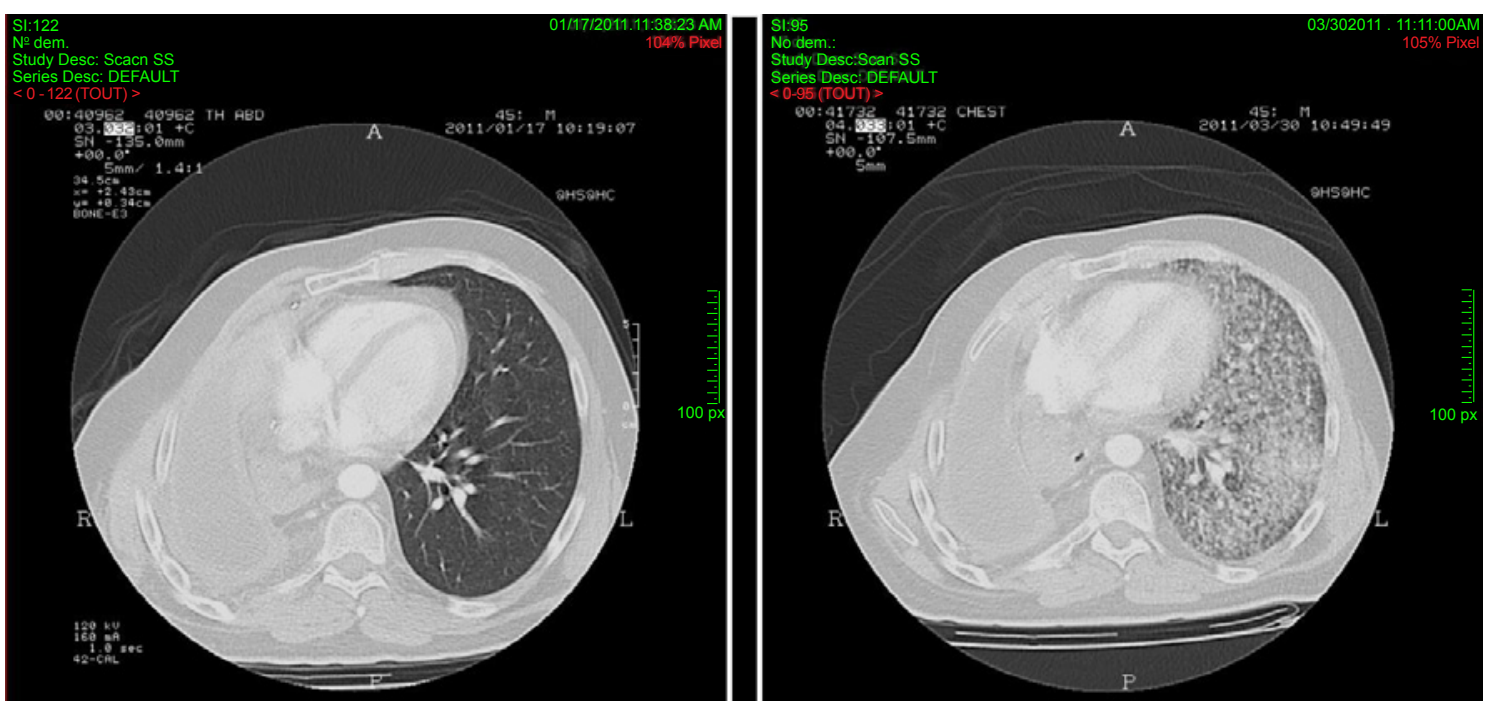

Figure 2: To the left: CT Scan of the chest done on 1/17/2011 (19 months post Right pneumonectomy) showing normal Left lung. To the right: CT scan after 2.5 months at the same level showing diffuse parenchyma infiltrates.

It is important to mention that our review of the literature was somewhat difficult because of the various descriptions and the nomenclature used in reporting this pattern of metastasis, such as: "extensive pulmonary metastasis" [2,13,14] "lymphangiitis carcinomatosis" $[8,12]$, "miliary pulmonary metastasis" $[3,5,10]$. Since the definition of carcinosis or carcinomatosis is the spread of the cancer to the whole body or the whole organ, we suggest naming this condition "pulmonary lymphangitic mesotheliomatosis" because the organ involved (pulmonary), the pattern of involvement (lymphangitic) and the kind of cancer (mesothelioma) are well defined.

\section{References}

1. Fishman AP, Fishman JA (2008) Fisherman's pulmonary diseases \& disorders $\left(4^{\text {th }}\right.$ edn), McGraw Hill Professional.

2. Sharif S, Zahid I, Routledge T, Scarci M (2011) Extrapleural pneumonectomy or supportive care: treatment of malignant pleural mesothelioma? Interact Cardiovasc Thorac Surg 12: 1040-1045.

3. Huncharek M, Muscat $J$ (1987) Metastases in diffuse pleural mesothelioma: influence of histological type. Thorax 42: 897-898.

4. Kaye JA, Wang AM, Joachim CL, Seltzer SE, Cibas E, et al. (1986) Malignant mesothelioma with brain metastases. Am J Med 80: 95-97.

5. Musk AW, Dewar J, Shilkin KB, Whitaker D (1991) Miliary spread of malignant pleural mesothelioma without a clinically identifiable pleural tumour. Aust N Z J Med 21: 460-462

6. Kim SB, Varkey B, Choi H (1987) Diagnosis of malignant pleural mesothelioma by axillary lymph node biopsy. Chest $91: 279-281$.

7. Fares M, Abbas O, Jamaleddine G, Bou-Khalil P (2008) Metastases in malignant pleural mesothelioma: a new radiological appearance. Respirology 13: 746-747

8. Craft PS, Reading MS, Jain S, O’Neil RA (1996) Lymphangitis carcinomatosa 
complicating primary malignant peritoneal mesothelioma. Thorax 51: 552553.

9. Ohishi N, Oka T, Fukuhara T, Yotsumoto H, Yazaki Y (1996) Extensive pulmonary metastases in malignant pleural mesothelioma. A rare clinical and radiographic presentation. Chest 110: 296-298.

10. Livasy CA, Tishko DJ, Maygarden SJ (2003) Miliary pulmonary metastases from a clinically occult pleural mesothelioma. Ann Diagn Pathol 7: 249-253.

11. Case records of the Massachusetts General Hospital. Weekly clinicopathological exercises. Case 23-1987. A 61-year-old woman with a left pleural effusion and a reticulonodular pulmonary infiltrate. N Engl J Med 316: 1462-1470
12. Nind NR, Attanoos RL, Gibbs AR (2003) Unusual intraparenchymal growth patterns of malignant pleural mesothelioma. Histopathology 42 150-155.

13. Uri AJ, Schulman ES, Steiner RM, Scott RD, Rose LJ (1988) Diffuse contralateral pulmonary metastases in malignant mesothelioma. An unusual radiographic presentation. Chest 93: 433-434.

14. Heki U, Fujimura M, Kasahara K, Matsubara F, Matsuda T (1999) Malignant mesothelioma presenting as pulmonary metastasis ahead of growth of primary tumour. Respirology 4: 279-281. 